\title{
Erratum to: Epidemiology of Dementia: The Burden on Society, the Challenges for Research
}

\author{
Frank J. Wolters and MA Ikram
}

Erratum to:

Chapter 1 in: Robert Perneczky (ed.), Biomarkers for Alzheimer's Disease

Drug Development, Methods in Molecular Biology, vol. 1750, https://doi.org/10.1007/978-1-4939-7704-8_1

The name of the Chapter 1 author had been inadvertently mentioned as M. Arfan Ikram.

This has been corrected and the name has been correctly updated to Ikram MA. 